\title{
Agriculture biologique et agriculture familiale au Brésil. Pour une inscription territoriale des agriculteurs écologistes
}

Organic agriculture and rural householders: for a territorial inscription of the ecologists farmers

Paulo Eduardo Moruzzi Marques et Tatiana Ferreira nobre de Lacerda

\section{OpenEdition}

\section{Journals}

Édition électronique

URL : http://journals.openedition.org/economierurale/2385

DOI : 10.4000/economierurale.2385

ISSN : 2105-2581

Éditeur

Société Française d'Économie Rurale (SFER)

Édition imprimée

Date de publication : 5 décembre 2009

Pagination : 55-66

ISSN : 0013-0559

Référence électronique

Paulo Eduardo Moruzzi Marques et Tatiana Ferreira nobre de Lacerda, « Agriculture biologique et agriculture familiale au Brésil. Pour une inscription territoriale des agriculteurs écologistes », Économie rurale [En ligne], 313-314 | Septembre - décembre 2009, mis en ligne le 05 décembre 2011, consulté le 01 mai 2019. URL : http://journals.openedition.org/economierurale/2385; DOI : 10.4000/ economierurale. 2385 


\section{Agricultures biologique et familiale au Brésil Pour une inscription territoriale des agriculteurs écologistes}

Paulo Eduardo MORUZZI MARQUES • Escola Superior de Agricultura Luiz de Queiroz, Departamento de Economia, Administração e Sociologia, Universidade de São Paulo, Brésil

Tatiana FERREIRA NOBRE DE LACERDA • Universidade Federal do Rio Grande do Sul, Développement rural, Brésil

\section{Introduction}

D ans la région sud du Brésil, dans l'État de Santa Catarina, un ensemble d'agriculteurs familiaux s'est engagé dans différentes stratégies de reproduction sociale associées au projet d'agriculture biologique mené par l'Association des agriculteurs écologiques de l'Encosta da Serra Geral (Agreco).

Grâce à l'implantation d'un projet de développement en partenariat avec diverses organisations $^{1}$, l'association en question favorise la construction de stratégies socioéconomiques alternatives pour les familles rurales, assises sur les bases « de l'agroécologie, de l'agro-industrie et de l'agrotourisme ». Ce projet et cette organisation sociale contribuent graduellement à façonner une représentation territoriale de l'Encosta da Serra Geral autour des idées de valorisation du savoir-faire des agriculteurs et de rénovation du rôle attribué à l'agriculture, en particulier en ce qui concerne la préservation environnementale, la qualité alimentaire et l'entretien du tissu social.

Le choix pour l'agriculture biologique s'est consolidé avec la fondation de l'Agreco, qui a permis de créer des conditions favorables, telles que la garantie du marché (grâce à un accord avec un réseau de supermarchés), l'accès aux services techniques et l'encouragement de la diversifi-

1. L'examen du cadre des espaces inter-institutionnel et intra-institutionnel concernant l'Agreco est développé par Lacerda (2005). cation des activités. Cette conversion à l'agriculture biologique a lieu dans le cadre d'un désenchantement concernant la production de tabac. À ce propos, il convient de préciser que la modernisation de l'agriculture dans la région s'est fondée principalement sur l'intégration agroindustrielle autour du tabac. Néanmoins, depuis le début des années 1990, la culture du tabac est de moins en moins vue comme une activité rentable à cause de la chute des prix, de l'augmentation des coûts de production, de l'intensification du travail dont souffrent les familles en raison de la migration des jeunes et de la disparition progressive de l'entraide entre voisins et de la croissance des problèmes de santé provoqués par le travail épuisant ${ }^{2}$ et par l'utilisation intense de pesticides. En effet, l'idée selon laquelle la production de tabac constitue la seule source de revenus pour les agriculteurs de la région recule, ouvrant certaines voies pour la mise en place de nouvelles alternatives chez les familles rurales.

À cet égard, cet article vise à examiner les motivations et les stratégies des familles au sein des exploitations agricoles de cette zone de l'Encosta da Serra Geral. Dans cette perspective, l'étude consiste également à mettre en évidence les ajustements dans les sphères productive et domestique des unités familiales de production, du fait de leur

2. La production du tabac exige beaucoup de maind'œurre au long de son cycle, surtout lors de la récolte et du séchage. 
adhésion au projet inspiré par des principes de l'agroécologie. À ce titre, il s'agit de discuter notamment de la place et de la transformation de l'activité agricole dans le cadre de l'implantation du projet de développement durable et territorial animé par l'association Agreco. Effectivement, ces mutations se répercutent sur la représentation concernant l'agriculture, le développement et le territoire, ce qui est également considéré dans ce travail.

Dans les stratégies de reproduction adoptées par les familles étudiées, on a pu considérer deux champs d'analyse : celui qui concerne l'ensemble d'activités agricoles et non agricoles ; celui qui se réfère à la construction d'une agriculture dont la fonction va au-delà de la production d'aliments.

Le premier champ nous renvoie au débat sur la pluriactivité et le second à celui sur la multifonctionnalité de l'agriculture. Ainsi, il apparaît que, dans les processus de gestion des exploitations étudiées, la reproduction des familles est assurée par l'articulation des activités agricoles et non agricoles, liées, dans les circonstances étudiées, à la mise en place d'une agriculture multifonctionnelle. En ce qui concerne l'analyse de ces exploitations agricoles, il nous semble approprié de concevoir que la reconnaissance de la multifonctionnalité de l'agriculture implique notamment de considérer les modifications de la nature du métier d'agriculteur (concernant l'invention et le développement de pratiques nouvelles) et de raisonner autrement sur les relations entre les exploitations et le territoire (Laurent et al., 2003).

En vue de contribuer à cette approche, ce travail analyse des exploitations agricoles du municipe de Santa Rosa de Lima, où se situe le siège de l'Agreco. À différents égards, ce municipe, situé à environ $120 \mathrm{~km}$ de Florianópolis, a été considéré comme représentatif des caractéristiques socioéconomiques, politiques et environnementales de l'Encosta da Serra Geral, région composée par seize municipes. En 2000,
Santa Rosa de Lima présentait 1584 habitants en millieu rural sur un total de 2007 (soit $73 \%$ ), selon l'Institut de planification et économie agricole de Santa Catarina (Paulilo et Schmidt, 2003). Par ailleurs, les exploitations agricoles du municipe sont, dans une grande mesure, considérées comme familiales (292 exploitations sur 303), avec une surface agricole moyenne de 42 ha (Incra, 2008) ${ }^{3}$. Dans ce cadre, Santa Rosa de Lima présentait, en 2002, le nombre le plus important d'exploitations agricoles biologiques (61) dans l'État de Santa Catarina, correpondant à $9 \%$ du total de cet État brésilien (Paulilo et Schmidt, op. cit.).

La fondation de l'Agreco a favorisé la mise en place d'un réseau de partenaires pour capter notamment des aides et des crédits issus des politiques publiques, permettant alors une dynamisation des interactions productives et socioéconomiques, ainsi qu'un renforcement des stratégies adoptées par les familles. Dans ce cadre, l'agriculture biologique s'associe à la constitution d'un réseau de petites agroindustries familiales (Projet intercommunal d'agro-industries modulaires en réseau), dont l'implantation ajoute de la valeur aux produits agricoles. Dans le même ordre d'idée, l'association Acolhida da Colônia, partenaire du réseau Accueil paysan, encourage l'agrotourisme et la valorisation de l'activité agricole.

Ces projets autour de l'agriculture biologique créent de nombreuses controverses compte tenu des conceptions distinctes de développement. Bien que l'Agreco soit un centre d'initiatives convergentes, d'autres projets de développement existent dans le territoire étudié, suivant principalement trois orientations : la croissance des

3. Incra, Novo retrato da agricultura familiar: $o$ Brasil redescoberto. Instituto nacional de colonização e reforma agrária, disponible sur : http://200.252.80.30/sade/creditos.asp dernier accès en juin 2008. 
surfaces reboisées, le maintien de la filière du tabac et le tourisme indépendant de l'association Acolhida da Colônia.

Quoi qu'il en soit, la mise en place du projet de développement mené par l'Agreco déclenche des transformations considérables dans les stratégies de reproduction sociale des familles rurales. Ces mutations concernent, d'une part, l'organisation du système productif et la gestion de l'exploitation et, d'autre part, la division de tâches au sein de la famille. En effet, l'analyse des stratégies de reproduction sociale des familles rurales s'inscrit dans le cadre des interrogations et des débats sur la persistance des exploitations familiales face au mode de production capitaliste. Cette continuité s'associe de plus en plus aux récentes transformations du marché du travail en milieu rural, marqué par la croissance des activités non agricoles.

Le concept de stratégie adopté permet de construire un lien entre les familles rurales et la société (y compris le territoire), mettant en évidence les notions de choix, d'interactions et de possibilités. Ce concept entraîne encore (selon Crow et Schneider, $o p$. cit.) une réflexion sur le type d'action, rationnelle ou non rationnelle, entreprise par ceux qui prennent des décisions. Dans cette optique, les stratégies reflètent les choix des individus, mais dépendent aussi de l'environnement extérieur. Par conséquent, l'analyse évite le piège de la dichotomie sociologique entre structure et agent ou entre société et individus.

Cet article, issu d'une étude plus élargie (Lacerda, op. cit.) a notamment utilisé les données obtenues grâce à la recherche mise en place par le dispositif Inra-Cemagref-Cirad $^{4}$ (Roux et al., 2004). Dans ce travail de recherche, l'équipe de l'État de Santa Catarina, sous la direction de Wilson Schmidt a, en 2002, interviewé vingt chefs d'exploitations adhérents à l'Agreco ${ }^{5}$. En 2004 et 2005, Tatiana Lacerda a réalisé des entretiens auprès de onze familles appartenant à l'échantillon précédent afin de mettre à jour les informations obtenues. Une enquête complémentaire a permis de les interroger sur d'autres aspects jugés pertinents pour cette nouvelle recherche.

Une interprétation des motivations et des stratégies de reproduction élaborées par les familles associées au projet de développement de l'agriculture biologique est présentée dans une première partie. La pluriactivité du chef d'exploitation et celle des membres de la famille sont mises en avant afin de mettre en évidence les nouvelles formes de fonctionnement des exploitations. En effet, la pluriativité particulière de cette zone contribue à une dynamique qui favorise l'identité du territoire, valorisant ainsi les rôles environnemental, social et culturel de l'activité agricole.

C'est dans cette perspective que la seconde partie de l'article développe des idées pour, d'une part, nourrir les débats sur la pluriactivité et la multifonctionnalité - comme celui de l'ensemble des questions que posent les frontières de l'activité agricole (Laurent et Rémy, 2004) - et, d'autre part, éclairer l'analyse de la réalité construite et vécue par les agriculteurs de la région de l'Encosta da Serra Geral.

4. Cette recherche financée dans le cadre du dispositif Inra-Cemagref-Cirad, s'articule autour de deux recherches antérieures. La première est le projet $\mathrm{CNPq}$ (Conseil national de la recherche scientifique) intitulé «Stratégies de développement rural, multifonctionnalité de l'agriculture et agriculture familiale : identification et évaluation des expériences dans diverses régions brésiliennes ", d'une durée d'un an (2002-2003). La seconde est le projet Capes/Cofecub dénommé «La notion de multifonctionnalité de l'agriculture, aspects analytiques et implications dans les politiques publiques : l'expérience française et le cas du Brésil ». Il s'agit d'un projet de coopération et d'échanges scientifiques associant les institutions brésiliennes impliquées dans le projet précédent avec l'Ina-PG, l'Inra et le CRBC/EHESS.

5. L'Agreco comptait 48 adhérents en 2005. 


\section{Les motivations et les stratégies des familles agricultrices}

En tant qu'associées au projet de développement animé par l'Agreco, les familles étudiées élaborent un ensemble de stratégies qui affirment l'identité de l'agriculteur comme producteur d'aliments et qui assurent une production (économique et sociale) autour du patrimoine familial.

Dans notre interprétation, trois aspects peuvent être retenus pour l'étude des stratégies des familles rurales représentatives. Nous avons notamment souligné les motivations qui orientent leurs choix et les changements de pratiques et de valeurs qui les accompagnent. Ces aspects se réfèrent aux choix concernant d'abord l'espace de développement écologique, puis la gestion de l'exploitation et de la main-d'œuvre familiale et, enfin, les activités purement agricoles et les occupations non agricoles.

\section{L'espace de développement écologique}

Ce choix se réfère à l'adhésion aux associations et au réseau de partenaires impliqués dans une démarche écologique. Cette adhésion et les initiatives de produire biologiquement, de réaliser la transformation des produits agricoles dans des petites agro-industries rurales et d'offrir des services touristiques s'associent à une stratégie territoriale dans la mesure où elle s'ancre dans la perspective de valorisation des spécificités du territoire (Pecquer, 2002). Il convient de souligner que, audelà de l'encouragement à la production biologique et aux activités non agricoles, l'association cherchera à forger l'identité culturelle du territoire, correlées par la cohérence entre les diverses interventions locales (Paulilo et Schmidt, op. cit.). Cet objectif est particulièrement conforté par l'Association Acolhida da Colônia, dont l'un des buts est de valoriser les fonctions sociale et productive de l'agriculteur.

À titre d'exemple, cette association a mis en place un projet d'agrotourisme englo- bant un ensemble d'activités développées par les familles paysannes. Selon cette logique, l'intention est de favoriser la pérennisation de l'activité agricole au sein des exploitations familiales, ce qui demande l'observation de certains principes :

- prédominance de l'activité agricole sur celle d'accueil à la ferme ;

- mise en valeur du patrimonie de l'exploitation agricole grâce à la restauration des bâtiments, en respectant le style original ; - mise en avant de l'expérience de vie des agriculteurs (concernant notamment leurs engagements en vue de la qualité des produits alimentaires et des services environnementaux) dans la publicité et les renseignements autour de cette initiative.

En somme, la mise en place des activités touristiques vise à favoriser la reconnaissance du mode de vie agricole grâce à une intensification de l'interaction sociale, en particulier, autour d'un dialogue sur la question environnementale.

Au-delà de 1'Associação Acolhida na Colônia, le réseau de partenaires de l'Agreco est composé d'une coopérative de professionnels et d'une autre de crédit, un centre de formation (Centro de Formação da Encosta da Serra Geral), un forum de débat sur le développement constitué par une autre association locale.

Dans ce cadre, il convient aussi de mentionner les principaux projets et programmes pris en compte par les familles rurales en vue de la mise sur pied de leurs stratégies de reproduction :

- Projeto Intermunicipal de Agroindústrias Modulares em Rede (Piamer), visant à promouvoir un large processus de développement solidaire dans la région grâce à la valeur ajoutée sur la production de l'agriculture familiale et à la création d'opportunités de travail et de revenus. Dans ce projet, 53 agro-industries à petite échelle ont été prévues, engageant 211 familles d'agriculteurs (Lacerda, op. cit.) ;

- Merenda Escolar, projet du gouvernement de l'État de Santa Catarina assurant l'ap- 
provisionnement en aliments bio des écoles publiques. Il s'agit du plus important acheteur de l'Agreco, correspondant à presque $50 \%$ des ventes de l'association ;

- Programa de Desenvolvimento da Agricultura Familiar Catarinense pela Verticalização da Produção, fondé sur un accord avec le Conseil national d'appui à la recherche scientifique $(\mathrm{CNPq})$ et la Fondation de science et technologie de l'État de Santa Catarina (Funcitec), permettant le recrutement de différents professionnels (agronomes, ingénieurs d'aliments et publicitaires, notamment) afin d'appuyer la mise en place des petites agro-industries ;

- Programa de Certificação em Grupo (Ecoccert) permettant l'obtention d'un certificat de conformité aux normes de l'agriculture biologique grâce à un système de contrôle collectif, représenté par l'association.

Les familles adhérentes à l'Agreco ont toutes choisi de participer à un nouvel espace qui diffère considérablement des cadres familial et communautaire auxquels elles appartenaient et appartiennent. Le sens attribué à ce nouvel espace se matérialise aussi bien dans l'implantation de nouvelles installations que dans les nouveaux rapports, basés sur des représentations et des conceptions dans une grande mesure inédite. Au-delà de l'espace physique de l'Agreco, les exploitations familiales constituent elles-mêmes de nouveaux espaces et des rapports également nouveaux autour des agro-industries à petites échelles, conçues dans la majorité des cas comme une extension de l'exploitation. Néanmoins, cela implique le regroupement familial, encouragé par l'Agreco et justifié, d'un point de vue structurel, par la fonctionnalité et le moindre coût de l'assistance technique. Cette solution s'associe également aux exigences de l'implantation du projet en faveur des agro-industries (projet Piamer), dont le financement a été assumé collectivement, étant donné le montant élevé de l'investissement nécessaire pour faire face non seulement aux normes sanitaires et techniques mais aussi pour l'échelle minimale de production.

De plus, quelques familles s'inscrivent dans l'espace mis en place par le projet agrotouristique, soutenu par l'association Acolhida da Colônia. Dans tous ces espaces, un nouveau cadre de valeurs et de perceptions germe autour des techniques productives et des questions environnementales, sociales et politiques.

Accomplissant des actions reconnues, l'association d'agriculteurs écologiques et la présence régulière de touristes passionnés par l'agrotourisme ${ }^{6}$ favorisent la propagation d'une conscience écologique exprimée dans la croissance du nombre d'agriculteurs qui adhérent à l'agriculture biologique ou, tout au moins, qui essaie de réduire l'utilisation de pesticides dans leurs exploitations? ${ }^{7}$. Cette tendance est surtout confortée par des expériences antérieures négatives, telles que le décès ou la maladie de l'un des membres de la famille ou en raison de l'insalubrité des exploitations du voisinage resté fidèle à la production du tabac.

Dans ce nouvel espace, les notions de paysage et de nature ne s'opposent pas à l'activité agricole, dont les effets maléfiques et concentrés sont imputés à la production de tabac et la dévastation des forêts par le feu.

\footnotetext{
6. L'agrotourisme peut être défini comme un tourisme orienté spécialement vers la valorisation des activités et des productions agricoles. Cette notion s'oppose à celle de tourisme rural ou écologique centré essentiellement sur l'idée d'une nature totalement protégée de la présence humaine (Diegues, 1996). Elle s'oppose également à celles associées au tourisme d'aventure qui est en cours d'implantation dans la région (rafting).

7. On notera parmi les témoignages des familles que, selon leur perception, l'agriculture qu'ils pratiquent contribue à l'entretien de la nature : «Parce qu'on n'utilise pas de pesticides (...) on pratique l'agroécologie (...) on ne brûle pas les bois et on n'utilise pas les traitements chimiques (...) on n'encourage pas la déforestation et on ne dévaste pas l'environnement (...) on préserve l'eau ».
} 
Cette confrontation identifie le conflit entre les exploitations liées à la filière du tabac et celles inscrites dans le projet de l'Agreco. Les exploitants-adhérents soutiennent une fonction agricole productive associée à la préservation de l'environnement, à la qualité alimentaire et à la culture locale. Par ailleurs, bien que le conflit entre «terre de travail » et « terre de préservation environnementale » ne se manifeste pas dans le cadre des projets de l'Agreco et de l'Acolhida da Colônia, d'autres services, récemment offerts dans le municipe de Santa Rosa de Lima (concernant le rafting et les eaux thermales), peuvent devenir une source de controverses, car dans ces cas, la nature est dissociée de l'activité agricole.

\section{Le travail familial}

Ce deuxième ensemble relatif aux motivations et aux stratégies suppose une réflexion sur les conceptions de l'agriculture familiale. En effet, il est important de concevoir cette structure comme une unité sociale qui se reproduit dans un régime d'économie familiale, dans laquelle les membres partagent un espace commun, possèdent l'accès à la terre et s'unissent grâce au lien de parenté (Maluf, 2002). Il s'agit d'une conception selon laquelle l'agriculture familiale constitue à la fois une unité domestique et productive, dont l'objectif capital consiste à assurer la reproduction sociale et économique du groupe grâce à l'élaboration de stratégies individuelles et collectives. Au sein des familles rurales, différents facteurs sont articulés afin de garantir la reproduction du groupe en tant qu'agriculteurs familiaux.

La volonté de reproduction de l'exploitation familiale est la première motivation des agriculteurs. Ainsi, bien que les membres de la famille exercent des activités variées et en dépit du fait que les familles de la région soient majoritairement petites (composées du père, de la mère et de deux enfants), la main-d'œuvre est fondamentalement issue du réseau familial. En cas de grand besoin, ces familles échangent des jours de travail entre parents et adhérents de l'association. En outre, la principale stratégie d'accès à la terre reste effectivement centrée autour du réseau familial.

Dans les agro-industries locales et dans les services touristiques, le travail est assuré par les membres de la famille, malgré l'introduction de nouvelles attributions et, parfois, l'éclosion de certains conflits en raison de la déstructuration de la hiérarchie familiale qui existait auparavant.

L'organisation des activités varie entre les agro-industries, pouvant être exécutées par un ou deux membres de chaque famille impliquée ou par seulement une partie de l'ensemble des familles, qui manipulent ainsi la matière première agricole provenant de toutes les exploitations concernées. Dans le cas des agro-industries familiales, les activités peuvent être assurées indifféremment par tous les membres. Il n'y a pas de division du travail dans les ateliers de transformation alimentaire, mais il faut souligner que les femmes jouent un rôle fondamental, s'occupant notamment de l'organisation des activités et du nettoyage des installations. Concernant les activités de pesage ou la comptabilité, on note que ce sont les jeunes plus scolarisés qui occupent ces fonctions. Dans les ateliers sous la responsabilité de plusieurs familles, le travail en coopération favorise une division des tâches de manière coordonnée où les femmes occupent une place reconnue. Quant à l'agrotourisme, on peut noter que le nombre des familles engagées est réduit. En effet, il s'agit d'une activité qui exige des changements considérables, s'agissant plus spécialement de l'aménagement de l'exploitation et, de nature plus difficile, des modes de vie (réduction du temps de repos dans la mesure où ces activités se réalisent surtout le week-end et élargissement des relations sociales, ce qui impose dans ce cas une posture très attentive par rapport aux pratiques en termes d'alimentation, d'hygiène, d'hospitalité et d'habillement). 
À tout cela, on peut encore ajouter des difficultés concernant la communication, l'infra-structure municipale (comme celle de la conservation des routes), le financement des investissements et l'affluence irrégulière de touristes.

De toute manière, les exploitations familiales, fournissant des services touristiques, connaissent des opportunités plus larges de travail, particulièrement pour les femmes et les jeunes. Ils s'occupent de l'administration de ces services permettant une augmentation des revenus familiaux, sans oublier la valeur ajoutée des produits de l'exploitation, consommés par les visiteurs. Par ailleurs, en termes de cohabitation sociale, il s'agit aussi d'un gain réel et considérable où apprentissage personnel et familial s'ajoutent à la reconnaissance du rôle de l'agriculteur, associée à la mise en valeur de son métier, de son exploitation et de ses actions pour la préservation de l'environnement.

Dans l'ensemble des exploitations étudiées, on observe, d'un côté, une proportion relativement significative de fils et de filles d'agriculteurs s'établissant sur l'exploitation et, d'un autre côté, le souhait des parents de voir leurs enfants perpétuer la profession au sein de la famille, habiter en milieu rural, préservant ainsi leur patrimoine. En somme, malgré la diversification des activités et des sources de revenus, ces exploitations agricoles demeurent ancrées dans les réseaux familiaux qui leur assurent l'accès à la terre et l'apport de forces complémentaires de travail, confortant de la sorte les attentes, les espoirs et les projets futurs.

\section{Les activités agricoles et non agricoles}

La majorité des membres des familles étudiées, adhérents à l'association, ont comme activité principale l'agriculture. Mais l'ensemble des activités non agricoles - agro-industrie familiale, agrotourisme, commercialisation directe - émanent de l'agriculture ou s'associent aux services domestiques. Dans une certaine mesure, ces familles peuvent être considérées comme pluriactives, car elles réalisent plus d'une activité et possèdent plus d'une source de revenus. Toutefois, il convient de souligner que les activités non agricoles effectuées par les familles sont mises en place au sein de l'exploitation et, pour cette raison, elles pourraient être désignées comme activités para-agricoles ( $c f$. infra).

Cette distinction permet d'affirmer que les activités para-agricoles ne se réfèrent ni à l'emploi refuge qui assure une certaine sécurité - constaté par Graziano da Silva (2001) dans la région sud-est du Brésil ${ }^{8}-$ ni aux activités industrielles étudiées par Schneider (2003) et Sacco dos Anjos (1995) dans le sud du pays. Même si l'on considère qu'une partie des activités des familles rurales en question relève de la transformation des produits agricoles, ce phénomène ne peut pas se comparer au processus d'expansion d'un marché dynamique de travail industriel auquel ces auteurs se réfèrent.

Par ailleurs, les autres activités effectuées par les familles adhérentes à l'Agreco sont issues d'une actualisation des idées autour de la famille, du territoire rural et de l'agriculture, qui sont profondément enracinées et valorisées. Grâce à l'association, ce processus est donc parallèle à la promotion d'activités comme le tourisme, l'agro-industrie rurale à petite échelle, la commercialisation, allant bien au-delà des activités productives agricoles traditionnelles. Dans cette perspective, on favorise des pratiques bénéfiques à l'environnement en même temps que l'agriculture est privilégiée en tant qu'utilisatrice du sol. Pour cette raison, l'opposition entre l'espace de consommation et l'espace de production est très peu observable, opposition constatée dans certains pays européens où le milieu rural et tout ce qui concerne la ruralité sont de moins en moins associés à un espace fondamentalement productif (Abramovay, 2003).

\footnotetext{
8. Dans le cas étudié, les agglomérations urbaines étaient distantes et difficiles d'accès.
} 
Lors des entretiens auprès des familles étudiées et dans le cadre des stratégies économiques, la production agricole est considérée, comme la principale source de revenus dans les exploitations familiales. Bien que les produits soient souvent transformés dans les agro-industries locales, pour la plupart des familles, l'activité principale qui rémunère et qui assure la reproduction sociale continue à être attribuée à l'agriculture elle-même. Une telle représentation permet de constater l'importance conférée à l'activité agricole, associée à l'affirmation et au souhait de reproduction de l'identité d'agriculteur, surtout en tant que producteur d'aliments.

La production destinée à la consommation familiale a été considérée comme source indirecte de revenus. En effet, si celle-ci n'existait pas et si l'on considérait la valeur de ces aliments acquis sur le marché, les dépenses familiales seraient telles que la reproduction des exploitations agricoles familiales serait menacée. Cette production engage tous les membres de la famille et intègre aussi bien une partie des produits commercialisés que d'autres aliments orientés exclusivement vers la consommation domestique. Malgré l'importance pour l'approvisionnement alimentaire de la famille, cette activité n'est pas vue comme prioritaire du fait qu'elle n'est pas reconnue comme production agricole et que ses produits ne sont pas non plus destinés au marché. À cet égard, le travail qu'il a fallu assumer n'est pas non plus reconnu comme tel. Il devient une occupation intégrée à celles qui sont réalisées dans le quotidien de la sphère domestique, associée à la famille et accomplie pendant le temps libre.

Néanmoins, certaines exploitations, surtout celles orientées vers la production de canne à sucre, se concentrent de plus en plus exclusivement sur cette production, abandonnant souvent d'autres cultures, destinées au marché ou à la consommation de la famille. Cette tendance peut principalement s'expliquer par la réduction d'une main-d'œuvre disponible dans ces exploitations et l'absence de techniques appropriées à l'agriculture biologique.

Dans ce cas, les exploitants ont choisi de diversifier leurs activités (production de cassonade et melado, une sorte de jus de canne) au détriment d'une production diversifiée. Deux conséquences découlent de ce choix :

- une augmentation des achats dans le marché local ou du troc d'aliments entre voisins (irrégulier en raison des saisons); - une dépendance par rapport à la commercialisation d'un produit clé. Dans certaines circonstances, cette dernière peut provoquer une grande instabilité dans les stratégies de reproduction de l'exploitation, mais, dans ce cas particulier, une certaine sécurité est obtenue grâce à l'industrialisation du produit (ce qui rend sa conservation plus longue) et grâce à l'action de l'Agreco qui assure, dans une certaine mesure, une stabilité de marché9.

Ainsi, on peut observer que la diversification des activités ne correspond pas forcément à une diversification de la production agricole. Dans l'exemple présenté ci-dessus, ce phénomène constitue surtout une réponse des exploitants face à la demande croissante des agro-industries en matière première.

De toute manière, cette diversification d'activités (où la transformation ou l'offre de services jouent un rôle plus important dans l'exploitation) ne conduit pas à une perte d'identité de l'agriculteur, au contraire. On a pu constater en effet que ces activités s'intègrent, en dernière analyse, dans le champ de la profession d'agriculteur, permettant d'ailleurs une augmentation de

9. L'association commercialise seulement des produits certifiés par l'Eccocert, principalement destinés aux institutions publiques. Une partie moins importante des produits est mise sur le marché grâce à des accords commerciaux. Enfin, une autre petite portion des produits est directement vendue au consommateur, notamment dans des marchés locaux et magasins spécialisés. 
l'estime de soi, grâce notamment à ces nouvelles occupations permettant la consolidation des moyens de pérennisation de l'exploitation.

\section{Multifonctionnalité Pluriactivité et activités para-agricoles}

Au Brésil, les débats sur la multifonctionnalité de l'agriculture font apparaître l'émergence de la pluriactivité. Il s'agit d'interrogations sur les similarités entre ces deux notions qui détournent, dans une grande mesure, la réflexion sur le sujet. Il est évident que les frontières entre ces notions sont peu claires.

Dans cette seconde partie de l'article, on propose d'éclaircir plus précisément ces deux termes. Ainsi, la pluriactivité et la multifonctionnalité de l'agriculture sont deux idées qui délimitent des perspectives différentes tant au plan de l'intervention des politiques publiques que l'interprétation des approches, à partir d'angles distincts sur l'agriculture et sur le monde rural. Pour développer ce raisonnement, on reprendra la notion d'activité para-agricole, favorisant la séparation entre ces deux notions.

En ce qui concerne la pluriactivité, à partir des élaborations du champ des sciences sociales, on peut constater que les auteurs engagés dans cette réflexion soulignent les stratégies de reproduction des agriculteurs familiaux dans le cadre de la modernisation de l'agriculture brésilienne (Schneider, 2002). Ces stratégies se développent afin d'ajuster les exploitations agricoles familiales aux circonstances socioéconomiques qui leur sont totalement défavorables. La pluriactivité ne constitue pas en soi une rupture avec les logiques productivistes qui favorisent la concentration foncière, la dégradation environnementale et l'exclusion sociale. Il s'agit plutôt d'une accommodation non confortable sur un terrain hostile.

L'élaboration du cadre de références autour de la notion de multifonctionnalité de l'agriculture se forge parallèlement au débat sur le développement durable (Rémy, 2005). À différents égards, la construction de cette notion vise à instituer des dispositifs de politique publique qui puissent transformer les logiques dominantes de production et de consommation dans le système agroalimentaire. Dans cette perspective, on essaie de considérer, sur un premier plan, les aspects sociaux et environnementaux de l'activité agricole.

Ainsi, les idées autour de la notion de multifonctionnalité de l'agriculture convergent vers une réorientation des politiques publiques. Il s'agit d'appuyer les agriculteurs afin qu'ils puissent développer des actions favorables à la préservation environnementale, à la cohésion sociale, à l'équilibre territorial, à la qualité des aliments, etc. Évidemment, l'application de politiques de cette nature exige une profonde révision des valeurs concernant la consommation et la production, impliquant notamment le refus du présupposé selon lequel «produire plus signifie forcément produire meilleur ».

Dans le champ d'études des processus sociaux en milieu rural, la notion de multifonctionnalité de l'agriculture peut favoriser une focalisation de l'analyse sur la mise en place de dispositifs institutionnels dirigés vers la promotion de fonctions environnementales et sociales de l'agriculture et du milieu rural. Effectivement, il s'agit d'examiner les outils de politiques publiques destinés au développement rural, forgés dans un champ de controverses autour de projets pour l'agriculture.

Dans la mesure où les idées autour de la multifonctionnalité favorisent une revalorisation de l'agriculture au niveau de ses dimensions sociales et environnementales, il convient de concevoir, avec quelques précautions, que la notion se réfère surtout à l'activité agricole à temps complet. Les activités agricoles doivent être, dans cette optique, vues d'une manière plus élargie, ce qui est concevable si l'on considère l'idée d'activité para-agricole. Alors, il s'agit de 
la multiplication des activités internes ou étroitement associées à l'exploitation agricole, comme c'est le cas des initiatives des agriculteurs écologistes de l'Encosta da Serra Geral.

Au Brésil, certaines études, très récentes, se réfèrent aux activités non agricoles réalisées dans l'exploitation au titre de paraagricoles, s'appuyant sur les suggestions du programme de recherche Arkleton Trust $^{10}$. Il faut préciser que cette idée n'est pas adoptée pour limiter la notion de pluriactivité, mais pour élargir le sens qui lui était précédemment attribué (emplois multiples ou activités agricoles à temps partiel).

De notre point de vue, il convient de définir les nuances de ce champ d'analyse, séparant les activités para-agricoles de celles considérées comme pluriactives. Ainsi, les activités effectuées autour des agro-industries familiales, des services d'agrotourisme ou des initiatives en faveur de l'environnement (à condition qu'elles soient associées à l'exploitation agricole familiale) peuvent être interprétés d'une façon plus appropriée si elles sont identifiées comme activités para-agricoles.

Dans cette perspective, la pluriactivité se limite à l'activité agricole à temps partiel réalisée par les agriculteurs dans le secteur industriel ou tertiaire pouvant présenter des opportunités de travail pour la population locale. Dans leurs études sur les agriculteurs français, des auteurs (Roux et Fournel, 2003 ; Revel et al., 2004) n'évoquent pas la pluriactivité dans les cas où les agriculteurs exercent leurs (diverses) activités à plein-temps sur l'exploitation. D'ailleurs, ces auteurs considèrent que, dans ces circonstances, la diversification des activités s'inscrit clairement dans le cadre de la multifonctionnalité de l'agriculture.

10. Ce programme de recherche constitue une référence dans les études sur la pluriactivité, concernant notamment 24 régions de l'Europe.

\section{Conclusion}

Les agriculteurs étudiés ont choisi de s'insérer dans un projet de développement orienté vers la production biologique et vers la transformation alimentaire dans des petites agro-industries découlant du groupement de plusieurs familles. L'analyse empirique nous permet de concevoir un processus de mise en place, dans le territoire en question, d'un projet de développement rural fondé sur l'idée d'assurer la reproduction économique et, en même temps, familiale des exploitations agricoles, conservant certaines significations culturelles autour de l'activité agricole. Il s'agit d'un processus de consolidation d'un projet qui articule divers aspects, sur les plans social, économique, politique et environnemental.

Dans ce contexte, malgré les difficultés et les conflits identifiés, les familles reconnaissent qu'elles s'intéressent et s'engagent de plus en plus à produire, à consommer et à offrir des aliments plus sains, diffusant l'idée du « respect de l'environnement ». Dans ce sens, à côté d'une rationalité économique (instrumentale, qui oriente les démarches productives), d'autres valeurs subjectives émergent dans une dimension symbolique, développées à partir de la pratique de l'agriculture biologique et de l'élargissement du convivium social. En effet, un tel phénomène gère un processus de valorisation personnelle grâce à la renaissance de la fierté d'être agriculteur.

De fait, l'affirmation de l'agriculture constitue un pilier fondamental pour la construction des stratégies de reproduction sociale des familles étudiées dans la mesure où l'agriculture répond à la demande d'emplois, à la garantie de sécurité alimentaire et au déclenchement des dynamiques territoriales. Par ailleurs, la cohésion du tissu social, la vitalité de l'économie locale et la préservation de l'environnement sont des fonctions attribuées à l'agriculture, défendues au sein des exploitations agricoles familiales. Dans ce contexte, l'adhésion à 
une association d'agriculteurs biologiques constitue une pièce essentielle.

En ce sens, les stratégies établies par les familles autour de l'Agreco et du projet de développement durable contribuent graduellement à définir une agriculture spécifique, multifonctionnelle. Paulilo et Schmidt (op. cit.) soulignent deux aspects de la multifonctionnalité de l'agriculture : l'aspect économique et social (exprimé, d'une part dans la valeur ajoutée et, d'autre part, dans la création d'emplois dans le cas d'une agriculture biologique, celle-ci étant plus intensive en termes de travail) et l'aspect environnemental et territorial (promu par l'établissement de réseaux et d'aménagements productifs favorables à la consolidation du territoire et par l'impact positif sur l'environnement).

Ce processus se développe grâce à l'attribution de valeur à d'autres fonctions de l'agriculture, au-delà de la production, considérant profondément les formes d'insertion des exploitations familiales dans le territoire et valorisant les pratiques qui ne sont pas immédiatement productives. Dans cette perspective, on peut concevoir l'agriculture selon deux dimensions : la dimension productive qui offre des aliments végétaux, animaux et transformés (destinés au marché ou à l'autoconsommation) et la dimension au-delà de la production qui englobe les activités valorisées récemment, telles que celles concernant la préservation des ressources naturelles, l'entretien des paysages, la qualité des aliments, ainsi que le renforcement de l'agriculture familiale grâce à la création d'emplois et à la dyna- misation du territoire. Ce modèle multifonctionnel de l'agriculture constitue, alors, une rupture avec les logiques productivistes, réconciliant la production avec les rôles environnementaux et sociaux de l'activité agricole (Moruzzi Marques, 2002).

Enfin, les motivations autour des stratégies examinées ci-dessus consolident une agriculture qui favorise la préservation de la qualité de l'eau et du sol, l'entretien des paysages en tant que nature et ruralité, la reproduction socioéconomique des familles (création d'emplois grâce à la diversification des activités agricoles et non agricoles, ainsi que la dynamisation locale du commerce, de l'industrie et des services) et la sécurité alimentaire (qualitative et quantitative). Par ailleurs, elles s'inscrivent dans le cadre du développement d'un type de pluriactivité, qui pourrait, comme on l'a suggéré auparavant, être mieux définie dans le champ des activités para-agricoles, marquées par l'élargissement des travaux dans l'exploitation qui demeurent très associés à l'agriculture.

En somme, cette conjugaison de motivations et de stratégies permet aux exploitations familiales de se reproduire en tant que productrices d'aliments, mais elles deviennent également productrices de biens non-matériaux, tels que l'environnement, le territoire ou la qualité alimentaire. Ainsi, l'enchaînement de ce processus favorise et renforce l'élaboration de stratégies orientées vers la multiplication d'activités dans l'exploitation et l'adoption de méthodes productives qui mettent en avant les questions environnementales et sociales. 


\section{RÉFÉRENCES BIBLIOGRAPHIQUES}

Abramovay R. (2003). O futuro das regiões rurais. Série Estudos Rurais, Porto Alegre: UFRGS.

Carneiro M.-J., Maluf R. (Org.) (2003). Para Além da produção: multifuncionalidade e agricultura familiar. Rio de Janeiro, CPDA/UFRRJ, NEAD e Mauad.

Diegues A.-C. (1996). O mito moderno da natureza intocada. São Paulo, HUCITEC.

Graziano da Silva J. (2001). Quem precisa de uma estratégia de desenvolvimento? Brasília, Textos para Discussão, $\mathrm{n}^{\circ} 2$, NEAD/CNDRS.

Incra (2008). Novo retrato da agricultura familiar: o Brasil redescoberto. Instituto nacional de colonização e reforma agrária, http://200.252.80.30/sade/creditos.asp

Lacerda T.-F.-N. (2005). "A unidade familiar $e$ as novas funções atribuídas à agricultura: o caso dos agricultores ecológicos do território da encosta da Serra Geral". Mémoire soutenu au Programa de PósGraduação em Desenvolvimento Rural, Porto Alegre: Universidade Federal do Rio Grande do Sul.

Laurent C., Maxime F., Maze A., Tichit M. (2003). Multifonctionnalité de l'agriculture et modèles de l'exploitation agricole. Paris, Économie Rurale $\mathrm{n}^{\circ}$ 273-274, p. 134-152

Laurent C., Rémy J. (2004). Multifonctionnalité, activités, identités. Les cahiers de la multifonctionnalité, $\mathrm{n}^{\mathrm{o}} 7$.

Maluf R.-S. (2002). "O enfoque da multifuncionalidade da agricultura: aspectos analíticos e questões de pesquisa. In Lima Dalmo M. de Albuquerque; Wilkinson J. (Org.). "Inovação nas tradições da agricultura familiar”, Brasília, CNPq/Paralelo 15.

Moruzzi Marques P.-E. (2002). Agriculture familiale et participation au Brésil: les conseils municipaux de développement rural du PRONAF (acteurs, intérêts et pouvoir). Paris III, la Sorbonne nouvelle, Thèse de doctorat soutenue à l'Institut des hautes études de l'Amérique latine.
Paulilo M.-I.-S., Schmidt W. (Orgs.) (2003). Agricultura e espaço rural em Santa Catarina. Florianópolis , UFSC.

Pecquer B. (2002). Qualité et développement territorial: l'hypothèse du panier de biens et de services territorialisés. Paris, Économie Rurale, n 261 , p. 37-49.

Rémy J. (2005). Um caminho sinuoso e semeado de espinhos. Os agricultores franceses: da especialização e intensificação da produção à multifuncionalidade e ao desenvolvimento sustentável. Rio de Janeiro: CPDA, Estudos Sociedade e Agricultura, vol. 12, fascículo 1.

Revel A., Roux B., Bonnafous P., Fournel. E. (2004). Les stratégies innovantes des exploitations-ménages diversifiées. Paris, Colloque SFER «Les mutations de la famille agricole : conséquences pour la politique publique ».

Roux B., Fournel E. (2003). Multifuncionalidade e emprego nos estabelecimentos rurais franceses: um estudo das zonas montanhosas de Languedoc Roussilon. In "Para além da produção: multifuncionalidade e agricultura familiar", Rio de Janeiro, CPDA/UFRRJ, NEAD e Mauad.

Roux B., Bonnal P., Maluf R. (2004). Analyse au niveau local de la prise en compte de la multifonctionnalité de l'agriculture au Brésil à partir d'un dispositif comparatif régional : rapprochement du cas français. Les cahiers de la multifonctionnalité, $\mathrm{n}^{\circ} 6$. Sacco Dos Anjos F. (1995). A agricultura familiar em transformação: o caso dos colonos-operários de Massaranduba (SC). Pelotas, Editora Universitária/UFPEL. Schneider S. (2002). A pluriatividade como estratégia de reprodução social. In "Estudos sociedade e agricultura", Rio de Janeiro, CPDA/UFRRJ, n ${ }^{\circ}$ 16, p. 164-184.

Schneider S. (2003). A pluriatividade na agricultura familiar. Porto Alegre, Editora da UFRGS. 